PAPER

\title{
Relationship between the summing-localization behavior and perceived width of sound image
}

\author{
Koji Abe*, Daisuke Sunada, Shouichi Takane and Sojun Sato \\ Faculty of Systems Science and Technology, Akita Prefectural University, \\ 84-4, Aza Ebinokuchi, Tsuchiya, Yurihonjo, 015-0055 Japan
}

(Received 22 April 2009, Accepted for publication 15 January 2010)

\begin{abstract}
We examined the relationship between the summing-localization behavior and perceived width of a sound image via two hearing experiments based on relative comparisons of the perceived directions to two sound stimuli. Two types of sound stimuli were employed. One stimulus, the "composite stimulus," was generated by two sound sources located in horizontal plane with time lag $(-3.0$ to $3.0 \mathrm{~ms})$ between them. The composite stimulus produced a perception of the summing localization or precedence effect. The other stimulus was generated by a single sound source as the reference for the perceived sound image. The first experiment (Exp. 1) investigated the relationship between the perceived direction of the composite stimulus and the time lag between the sound sources. The second experiment (Exp. 2) was carried out in order to compare the perceived width of the composite stimulus to that of the reference stimulus. The results of Exp. 1 demonstrated that the perceived direction of the composite stimulus smoothly shifted from the middle of the sound sources to that generating the preceding sound, these findings roughly corresponded to the results of the past studies. However, the tendency of the shift was different among the subjects, and could be classified into two types according to whether the shift reached the direction of the preceding source. The results of Exp. 2 showed that the "included range," defined as the range for which the direction of the reference stimulus is perceived as included within the fused image of the composite stimulus, differed for each subject. The maximum width of the included range was over 30 degrees. From the results of Exps. 1 and 2 taken together, it is clear that there are individual differences with respect to how direction of a stimulus is perceived, within the included range of the fused sound image. This implies that the difference in the answer policy of each subject makes this difference.
\end{abstract}

Keywords: Summing-localization, Precedence effect, Direction and width of a fused image

PACS number: 43.66.Qp, 43.66.Lj [doi:10.1250/ast.31.260]

\section{INTRODUCTION}

Perception of sound image(s) produced by multiple sound sources is more complex than perception of images generated by a single sound source. For simplicity, we consider the case of two sound sources producing similar sound stimuli. In this case, three types of sound images can be perceived, according to the time lag between the sound sources. If the time lag is long enough, the sound stimuli from the two sound sources are individually perceived. This phenomenon is known as 'echo.' The echo threshold changes greatly from $2 \mathrm{~ms}$ to $100 \mathrm{~ms}$, as a function of the type of sound stimulus (e.g., click, broadband noise and or speech), as well as by the definition adopted as the echo threshold by different research groups [1-3]. In the case of

*e-mail: koji@akita-pu.ac.jp a time lag shorter than the echo threshold, the sound stimuli from two sound sources generate a single sound image, a "fused image" $[4,5]$. When the time lag is very small, the perceived direction of a fused image is decided depending on a weighted averaging of the information from both sound sources. This phenomenon is called 'summing localization.' As the time lag increases, a fused image gradually shifts toward the direction of preceding sound source. The shift to the direction of preceding sound source is completed at a lag of $0.63-1 \mathrm{~ms}[6,7]$. For time lags between this value and the echo threshold, the perceived direction of the fused image depends only on information from the preceding sound source. This specific localization phenomenon is called the 'precedence effect' [8-10].

In studies of sound localization, the perceived direction of the sound image is often subjectively answered. However, it is well known that localization is more vague 
for a fused image than a single sound source $[3,11]$. Nevertheless, little is known about the relationship between the perceived direction of a fused image and its width for a certain subject. To clarify the behavior of localization of a fused image produced by two sound sources with intervening time lag, it is necessary to measure the perceived direction of the fused sound image and its width at the same time. In order to investigate this relationship, we conducted two experiments based on a relative comparison between sounds generated by single and double sources. In this paper, a sound image generated by a single source was compared with a fused image generated by two sources, assuming that the sound image from a single source is localized in a direction corresponding to its position with small dispersion. The relative comparison enabled the perceived direction to be estimated statistically.

\section{EXPERIMENT 1 - DETERMINATION OF PERCEIVED DIRECTION AS A FUNCTION OF THE TIME LAG BETWEEN TWO SOUND SOURCES}

The purpose of Experiment 1 was to determine the relationship between the perceived direction of a fused sound image and the time lag between two sound sources. For this purpose, we adopted a relative comparison of the perceived direction produced by two types of sound stimuli. One stimulus was generated by two sound sources located in the horizontal plane with a time lag $(-3.0$ to $3.0 \mathrm{~ms}$ ) between them, producing a fused sound image with a perceived position that shifted from a position in between the sound sources and the location of the earlier source. This type of the sound stimuli is hereinafter called the "composite stimulus." The other stimulus was generated by a single sound source, and served as the reference for the perceived sound image, it is therefore, called the "reference stimulus."

\subsection{Method}

Experiment 1 was conducted in an anechoic room with dimensions as follows: $\mathrm{W}: 7.5 \mathrm{~m} \times \mathrm{D}: 6.0 \mathrm{~m} \times \mathrm{H}: 4.5 \mathrm{~m}$. Each subject sat on a chair at the center of the room. Thirteen loudspeakers were $3 \mathrm{~m}$ from the subject (see Fig. 1). These loudspeakers were arranged in directions from -55 to 55 degrees at 10 degrees interval ( 0 degree corresponds to the front of the listener). The two loudspeakers located at \pm 55 degrees were visual dummies; no sound stimulus was generated by these loudspeakers.

In each trial, a pair of sound stimuli, consisting of the composite and the reference stimuli, was presented. Each stimulus was presented only once, not repeated. The composite stimulus was always presented using two loudspeakers located at -45 and 45 degrees. The time delay set between these sound sources was defined as the

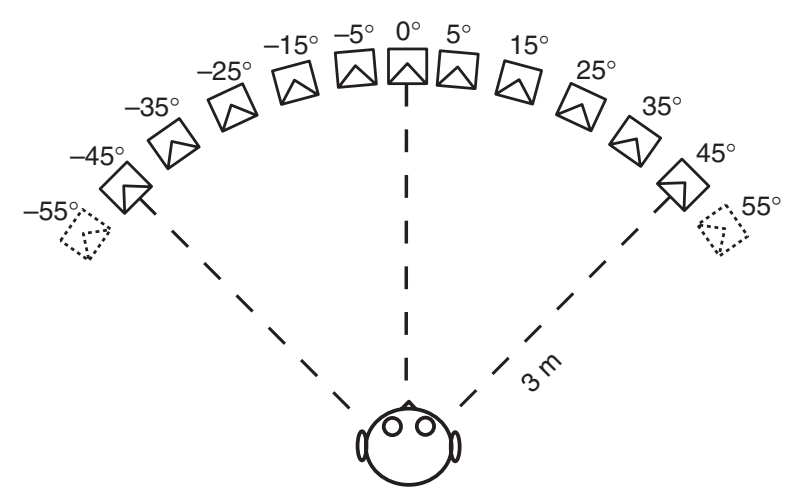

Fig. 1 Arrangement of loudspeakers for Exp. 1.

Table 1 Time lag between two loudspeakers and the corresponding number of trials set for Exp. 1.

\begin{tabular}{ccccccc}
\hline Time lag [ms] & 0 & \pm 0.1 & \pm 0.2 & \pm 0.3 & \pm 0.4 & \pm 0.5 \\
\cline { 1 - 6 } Number of trials & 10 & 10 & 10 & 10 & 10 & 10 \\
\hline Time lag [ms] & \pm 0.6 & \pm 0.7 & \pm 0.8 & \pm 0.9 & \pm 1.0 & \pm 1.2 \\
\hline Number of trials & 10 & 10 & 10 & 6 & 6 & 6 \\
\hline Time lag [ms] & \pm 1.4 & \pm 1.6 & \pm 1.8 & \pm 2.0 & \pm 3.0 & \\
\cline { 1 - 5 } Number of trials & 6 & 6 & 6 & 6 & 6 &
\end{tabular}

time lag. The time lag defined was positive when a sound from the loudspeaker at 45 degrees precedes that from the loudspeaker at -45 degrees. The time lags set for Exp. 1 are shown in Table 1, along with the corresponding number of trials. The reference stimulus was generated by one of the loudspeakers. Subjects were asked to answer whether the perceived direction of the latter stimulus was to the right or left of the former stimulus. The order of presentation of the composite and the reference stimuli was randomized. The A-weighted sound pressure level of the sound stimulus was $60 \mathrm{~dB}$ at the center of subject's head. The subject's head was not immobilized. A woman's speech with a duration of about $0.85 \mathrm{~s}$ was used for the source signal (/bakutiku/ in Familiarity-controlled Wordlists 2003 [12]). Ten untrained young college students who were compensated for their time (ages: 19-23) participated in this experiment. All subjects had normal hearing. Subjects listened to each stimulus and made his/her response to it in an initial pilot run in the presence of the experimenter. Pilot trials continued until the experimenter determined that the subject understood the task.

\subsection{Results and Discussion}

Figure 2 shows a typical experimental result for a time lag of $0.6 \mathrm{~ms}$. The abscissa shows the direction of the loudspeaker used to present the reference stimulus. The ordinate indicates the ratio answered: "The perceived 


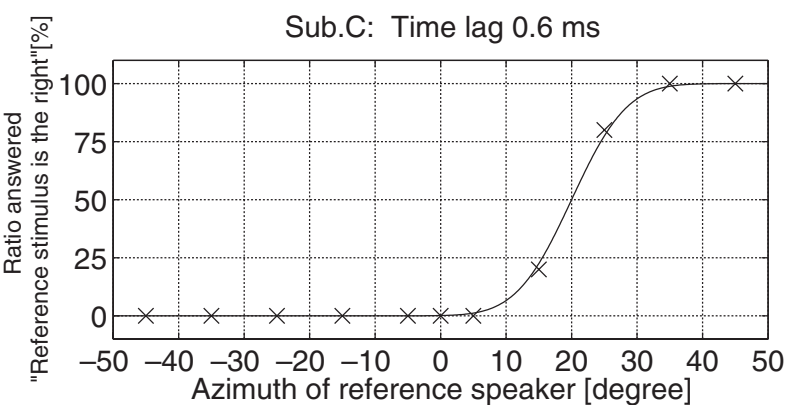

Fig. 2 Example of relationship between the ratio of leftright judgment of sound image and the direction of reference stimulus (time lag: $0.6 \mathrm{~ms}$ ). The reference speaker is a single speaker that generated the reference sound used for comparison with the composite sound image.

direction of the reference stimulus is right of that of the composite stimulus." Symbols " $x$ " in the figure designate the ratio obtained from the subject's answers. A cumulative normal distribution has been fitted to these data. The mean and a standard deviation of the fitted curve reflect the average and the ambiguity, respectively, of the "perceived direction." The ambiguity obtained from the fitting curve contains both the ambiguity produced by the composite and reference stimuli. The standard deviation obtained by the fitting curve in Fig. 2 is 6.6 degree. However, a sound image perceived by a single sound source can be ignored, because the difference in the sound source direction is much smaller than this deviation $[13,14]$. Therefore, the ambiguity obtained from Exp. 1 may result almost entirely from the composite stimulus.

After the same processing is applied to every value of time lag, the obtained mean directions are plotted against time lag as shown in Fig. 3, together with the corresponding standard deviation (error bars). Figure 3 shows the relationship between the perceived direction of a fused sound image and the time lag set between two sound sources ( \pm 45 degrees). The dashed lines in this figure indicate the positions of two loudspeakers presenting the composite stimulus.

Figure 3 shows that as the absolute value of the time lag increases, the fused sound image is shifting smoothly from 0 degrees to the direction of the preceding loudspeaker $( \pm 45$ degree) for almost all subjects. To determine the time lag at which the shift of the perceived direction toward the direction of the precedence sound source is complete, we set a nominal boundary of \pm 35 degrees. This value corresponds to the direction of the loudspeakers closest to the ones used to generate the composite stimulus. According to this nominal boundary, the shift of the perceived direction occurs between $0.4 \mathrm{~ms}$ and $0.9 \mathrm{~ms}$, except for Subjects I and J. Our results roughly correspond to the results of past studies $(0.63-1 \mathrm{~ms})[6,7]$.
Each panel in Fig. 3 indicates that the perception of subjects can be classified into two types, according to whether the average direction of the perceived sound image shifts up to the boundary ( \pm 35 degrees). All subjects except Subjects I and J completely shift the direction of the fused sound image to the preceding loudspeaker. On the contrary, Subjects I and $\mathbf{J}$ do not completely shift the direction of the fused sound image to the preceding loudspeaker.

\section{EXPERIMENT 2-INVESTIGATION ON PERCEIVED WIDTH OF SOUND IMAGE}

Experiment 2 was carried out in order to determine the directional range of a composite stimulus composed of two sound sources. Although this range is a little different from the width of the fused sound image, the value of the range can be taken to be similar to the width of the fused sound image, as described in Subsection 3.1. If the range can be obtained, the relationship between the answered direction in Exp. 1 and the width of the fused sound image can be specified for each subject. In order to allow direct comparison with Exp. 1, the same types of sound stimuli were used in Exp. 2.

\subsection{Method}

The experimental conditions were almost the same as Exp. 1. The reference stimulus, however, was presented from one of a set of loudspeakers located at 0 to 45 degrees relative to the subject, at intervals of 5 degrees (using only the loudspeakers in the right hand side of Fig. 1, and using five loudspeakers added to position of 10, 20, 30, 40 and 50 degrees). The time lag between loudspeakers in the directions of -45 and 45 degrees was always positive, and its upper limit was $1 \mathrm{~ms}$ (see the time lag in Table 2) because the shift to the direction of preceding loudspeaker was almost completed within this range for all subjects. Five trials were conducted for each condition. The reference sound stimulus was only presented on one side because the perceived direction of the fused sound image in Exp. 1 was almost symmetric with respect to the time lag.

In each trial, a pair of sound stimuli, consisting of the composite and the reference stimuli, was presented as in Exp. 1. The order of presentation, however, was fixed: the former was the composite stimulus, and the latter was the reference stimulus. Subjects were asked whether the sound

Table 2 Time lag between two loudspeakers and corresponding number of trials set for Exp. 2 .

\begin{tabular}{ccccccc}
\hline Time lag [ms] & 0 & 0.1 & 0.2 & 0.3 & 0.4 & 0.5 \\
\hline Number of trials & 10 & 10 & 10 & 10 & 10 & 10 \\
\hline Time lag [ms] & 0.6 & 0.7 & 0.8 & 0.9 & 1.0 & \\
\hline Number of trials & 10 & 10 & 10 & 10 & 10 & \\
\hline
\end{tabular}




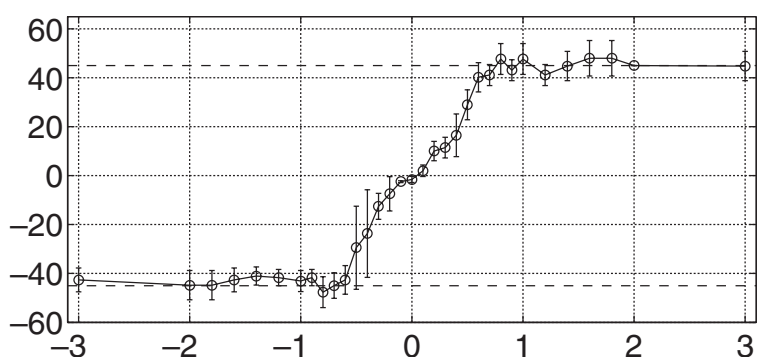

(a) Subject A

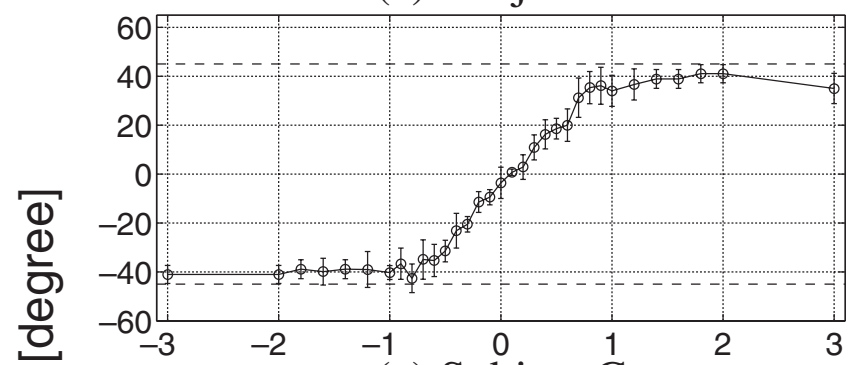

(c) Subject C

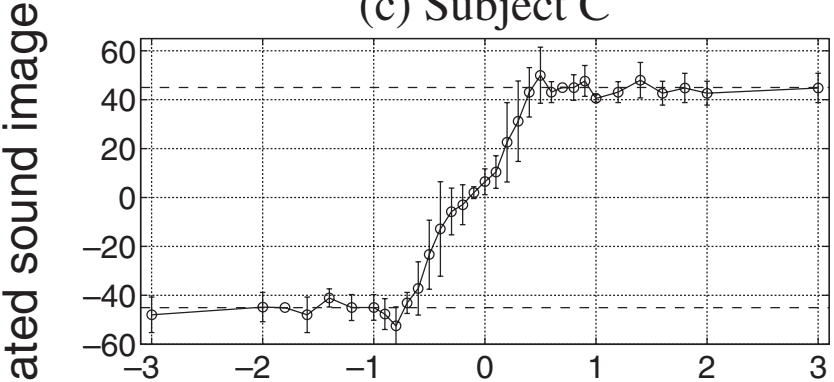

(e) Subject E

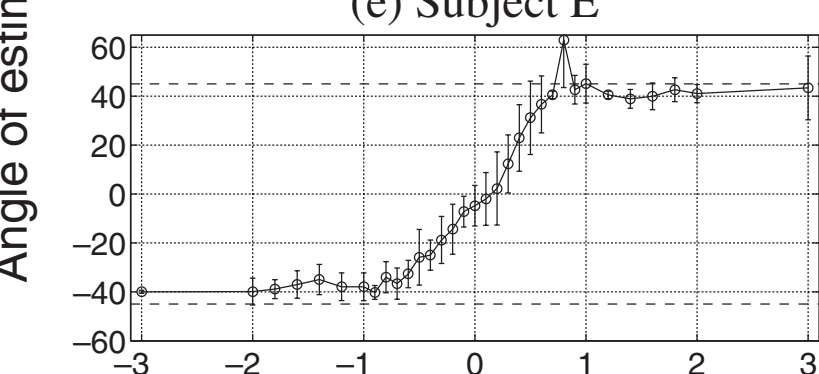

(g) Subject G

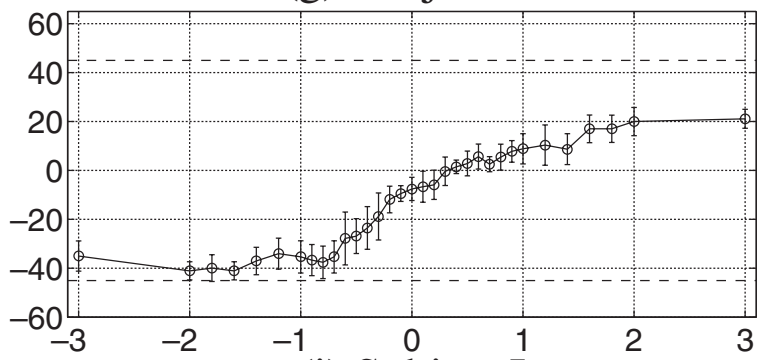

(i) Subject I

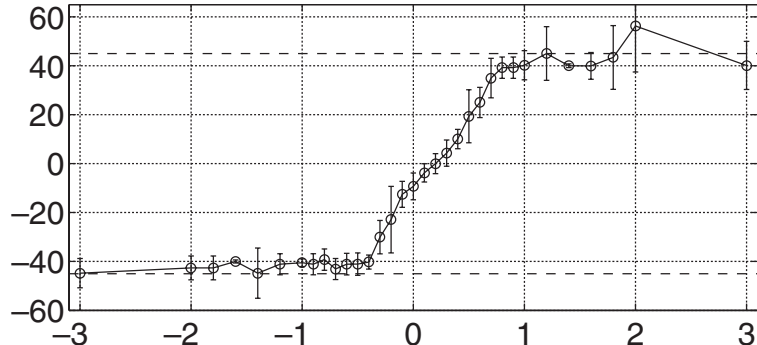

(b) Subject B

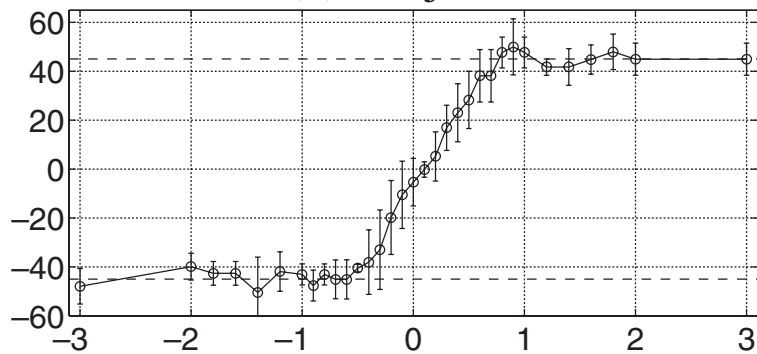

(d) Subject D

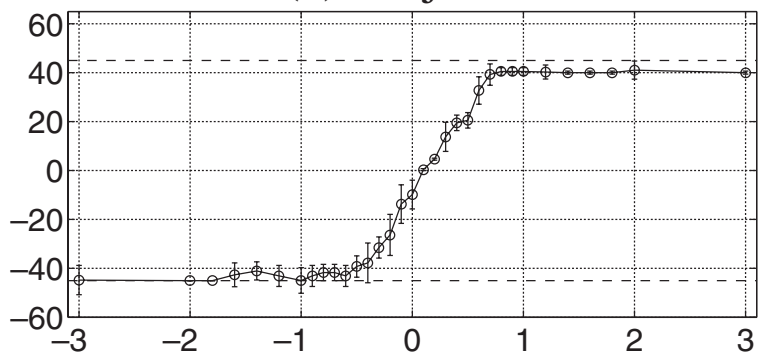

(f) Subject F

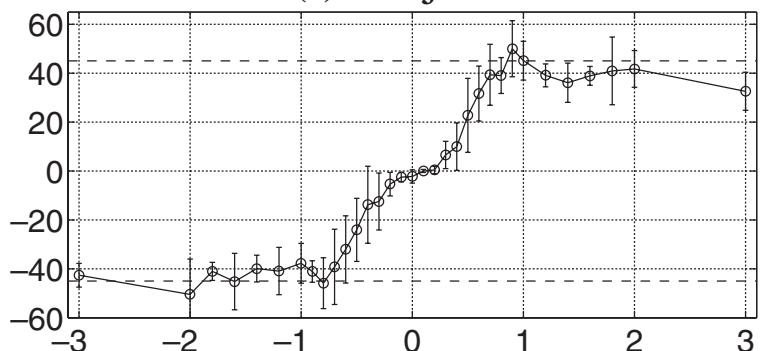

(h) Subject H

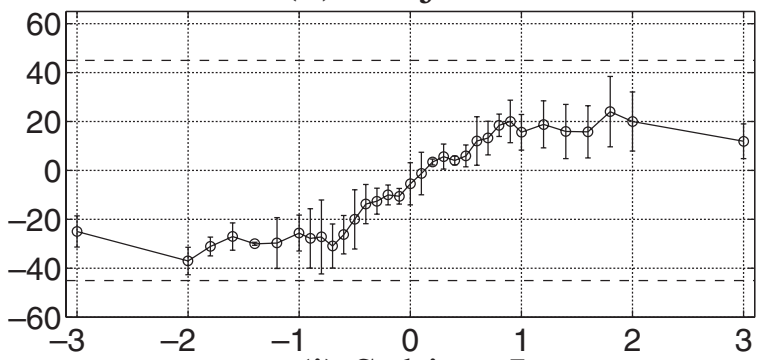

(j) Subject J

\section{Time lag $[\mathrm{ms}]$}

Fig. 3 Estimated direction of the fused sound image of the composite stimulus for each subject. Each panel shows the relationship between the time lag and the perceived direction of the fused sound image. ' $\bigcirc$ ' in the panels shows the mean $(\mu)$ of the fitted cumulative normal distribution, and the error bars indicate the corresponding standard deviation $(\mu \pm \sigma)$.

image of the reference stimulus was included within the width of the fused image produced by the composite stimulus. The subjects were seven young students who also participated in Exp. 1 (Subjects A-F and J).
When the fused sound image perceived by the composite stimulus spreads outside of the arranged loudspeakers in this experiment, the width of the fused sound image cannot be obtained accurately. Therefore, the range 
obtained from Exp. 2 is in the direction of a loudspeaker included in the width of fused sound image. Here, we call this range the "included range." Included range is less than or equal to the width of the fused sound image.

\subsection{Results and Discussion}

The results of Exp. 2 are shown in Fig. 4. The abscissa shows the time lag provided for the composite stimulus. The ordinate indicates the angle of the loudspeaker used to present the reference stimulus. The color bar indicates the ratio of the answers that the sound image of the reference stimulus is included inside that of the composite stimulus. The lines in Fig. 4 are the plots of the results of Exp. 1. The white rectangles on each time lag show the region where the "included" response is over $70 \%$, i.e., a ratio of $4 / 5$ or $5 / 5$. This range is the "included range" of each time lag. The included range is defined from the minimum angle to the maximum angle of the loudspeaker for which the answer ratio was over $70 \%$.

From each panel in Fig. 4, the stability of the subject's answer in this experiment can be seen according to the color of the cells. If the included range is fixed, the cells in the panel are painted in dark (corresponding to a ratio $>0.7$ ) or light (ratio $<0.1$ ) colors, and the region in each color is clearly separated. On the contrary, if the subject's answer is unstable, the number of cells in an intermediate (gray) color increases, and the included range does not obviously appear. As shown in Fig. 4, the included ranges for each subject are not compact compared with the interval of the arranged loudspeakers. The maximum widths of the included ranges in each subject are 30 degrees or more. The included ranges of Subjects A-E shift to the direction of preceding loudspeaker as the absolute value of the time lag increases. Consequently, the sound image of the front loudspeaker ( 0 degrees) is not included in the included range when the time lag is $1 \mathrm{~ms}$. On the contrary, the included ranges of Subjects F and J gradually spread to the direction of the preceding loudspeaker as the absolute value of the time lag increases.

As shown in Fig. 4(a), the fused sound image that Subject A perceives is relatively compact among all subjects (the maximum width of included range is about 30 degrees), and the perceptual judgment concerning the sound image is most stable. Stability means that a ratio of $70 \%$ or more exists, and the areas of high and low ratio are divided comparatively clearly. Although the maximum width of the included range for Subject $C$ is similar to that of Subject A. Figure 4(c) shows that the judgment concerning perceived sound image seems a little unstable because number of the cells of the high ratio is less than that of Subject A. Subject F is a subject who perceives the widest sound image (the maximum width of included range is about 55 degree). A similar tendency is also observed for
Subject J. These results suggest that the tendency to spread the fused sound image is not identical for each subject.

\subsection{Comparative Discussion of Exps. 1 and 2}

For Subjects A, B, D, F, and J, the means of the perceived direction of fused sound image obtained in Exp. 1 fall within the included range. In Subjects $\mathrm{C}$ and $\mathrm{E}$, there are some disagreements. As shown Fig. 4(c), the combination of the time lag and the direction of the loudspeaker in which the ratio of answers is $70 \%$ or more in Subject C is few, because his/her answers to the experimental tasks were unstable. As a result, we conclude that the mean of the perceived direction of the fused sound image is not in the included range. For Subject E, the mean of the perceived direction of the fused sound image deviates from the included range when the time lag is $0.2-0.5 \mathrm{~ms}$. This might be related to low answer accuracy about the perceived direction of the fused sound image in Exp. 1 (the length of error bar is about 30 degrees). Generally, there exists some correspondence between the perceived direction of the fused sound image and the included range.

In Exp. 1, some subjects reported perceived directions within the included range (Subjects A, B, D, F, and J). However, some of these subjects still exhibit differences with respect to where the perceived directions obtained in Exp. 1 are located within the included range obtained in Exp. 2. Subject A perceives almost exactly the center of the included range as the direction of the fused sound image. Subjects B and J exhibit a similar tendency, even though the reported direction shifts to the front loudspeaker. On the contrary, when the time lag increases and the sound image spreads, Subject F shows a tendency to report the area in the vicinity of the boundary of the included range as the direction of the fused sound image. Subject $E$ also have a similar tendency. These results show that there exist individual differences concerning the answer policy regarding which direction within the included range is decided as a perceived direction of the fused sound image. Specifically, the included ranges of Subjects $\mathrm{F}$ and $\mathrm{J}$ obtained from Exp. 2 are similar. However, for Subject J, the perceived direction of the fused sound image does not completely shift to the preceding loudspeaker, whereas the direction obtained for Subject $F$ shifts to the preceding loudspeaker. This reflects the difference in the answer policy.

\section{CONCLUDING REMARKS}

In order to examine the relationship between the main perceived direction of a fused sound image and its width, two experiments were conducted. From Exp. 1, we found that the perceived direction of the fused sound image smoothly shifted from the subject's front ( 0 degree) to the direction of the preceding loudspeaker as the absolute 


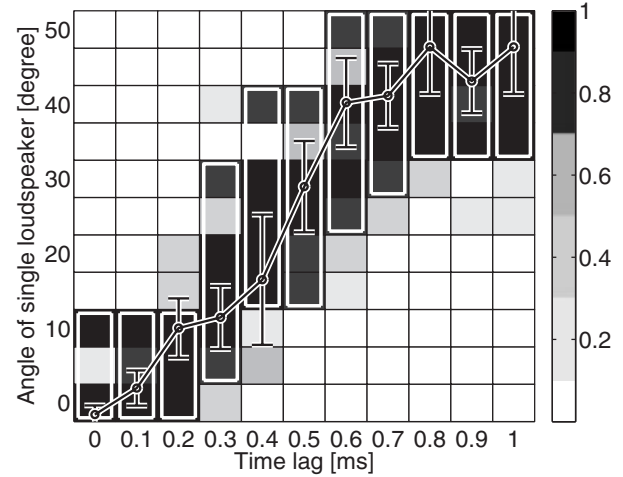

(a) Subject A

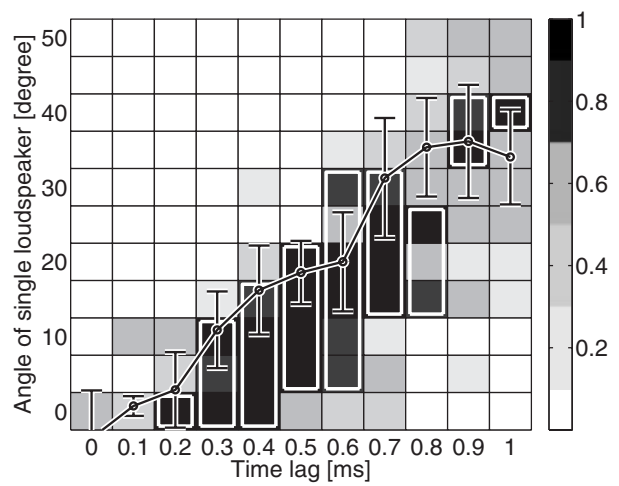

(c) Subject C

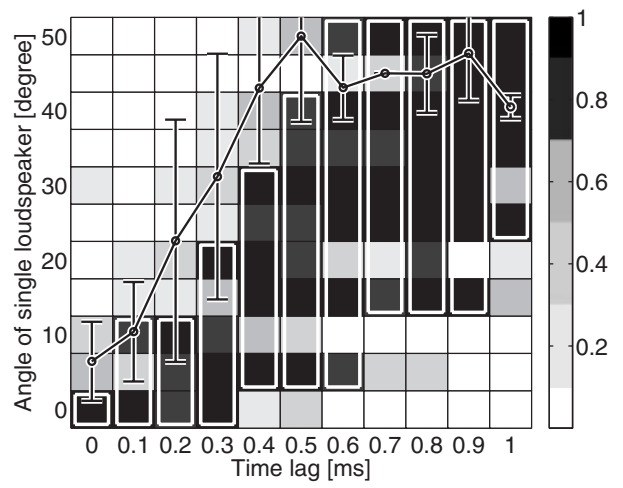

(e) Subject E

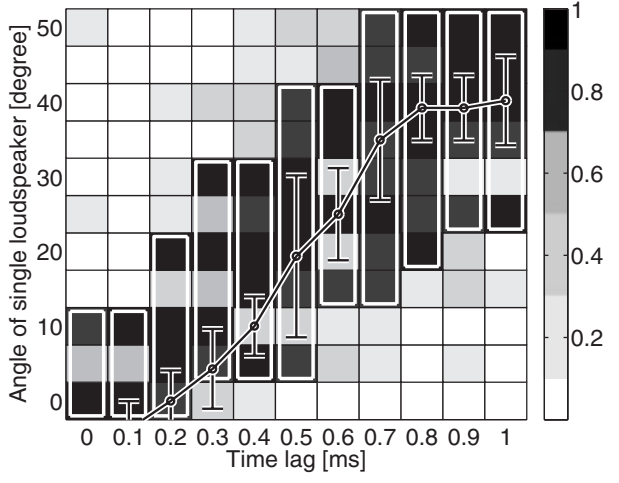

(b) Subject B

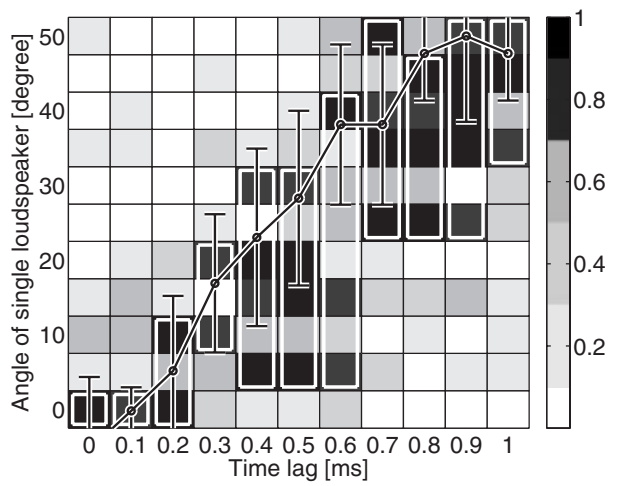

(d) Subject D

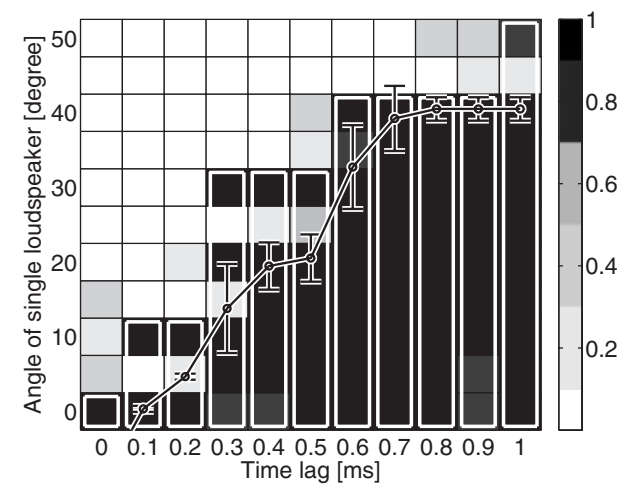

(f) Subject F

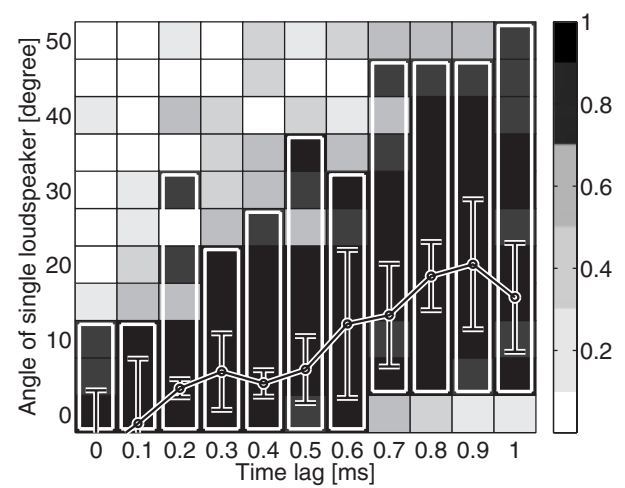

(g) Subject G

Fig. 4 The direction of the loudspeaker included in the width of the perceived sound image of the composite stimulus. The shaded region in each panel shows the ratio that the sound image of reference stimulus is perceived inside that of composite stimulus. The color-bars at the right side of each panel indicates the ratio as the density. The height of rectangles with thick white lines in each panel shows the "included range" at each time lag. 
value of the time lag increased. However, in some subjects, the perceived direction of the fused sound image did not completely shift in the direction of the preceding loudspeaker. Our results suggested that a time lag of $0.4-0.9 \mathrm{~ms}$ is necessary for completing the shift of the direction of the fused sound image to the preceding loudspeaker. This value corresponded to the result of previous studies concerning the precedence effect. In Exp. 2, we obtained the ranges in the direction of the loudspeaker included in the width of the fused sound image, called the "included range" in this paper. These included ranges were different among the subjects; the maximum value of include range was over 30 degrees. Taking the results of Exps. 1 and 2 together, we conclude that there is an obvious individual difference, regarding which direction is perceived within the included range of the fused sound image. Not only the perceived direction of the fused sound image, but also its width, must be taken into account when considering sound localization with multiple sound sources.

\section{REFERENCES}

[1] M. R. Rosenzweig and W. A. Rosenblith, "Some electrophysiological correlates of he perception of successive clicks," J. Acoust. Soc. Am., 22, 878-880 (1950).

[2] E. D. Schubert and J. Wernick, "Envelope versus microstructure in the fusion of dichotic signals," J. Acoust. Soc. Am., 45, 1525-1531 (1969).

[3] J. Blauert, Spatial Hearing: The Psychophysics of Human Sound Localization (MIT Press, Cambridge, Mass., 1983).

[4] V. L. Jordan, "A system for stereophonic reproduction," Acustica, 4, 36-38 (1954).

[5] D. M. Leakey, "Some measurement of the effects of interchannel intensity and time differences in two channel soud systems," J. Acoust. Soc. Am., 31, 977-986 (1959).

[6] J. Blauert and W. Cobben, "Some consideration of binaural crosscorrelation analysis," Acustica, 39, 96-104 (1978).

[7] J. Blauert, "Localization and the law of the first wavefront in the median plane," J. Acoust. Soc. Am., 50, 466-470 (1971).

[8] H. Haas, "Über den Einfluß eines Einfachechos auf die Hörsamkeit von Sprache [On the influence of a single echo on the intelligibility of speech]," Acustica, 1, 49-58 (1951).

[9] R. Y. Litovsky and B. G. Shinn-Cunningham, "Investigation of the relationship among three common measures of precedence: fusion, localization dominance, and discrimination suppression," J. Acoust. Soc. Am., 109, 346-358 (2001).

[10] K. Saberi and J. V. Antonio, "Precedence-effect thresholds for a population of untrained listeners as a function of stimulus intensity and interclick interval," J. Acoust. Soc. Am., 114, 420-429 (2003).

[11] K. Wendt, "Das Richtungshöern bei der Überlagerung zweier Schallfelder bei Intensitäts und Laufzeitstereophonie [Direc- tional hearing with two superimposed sound fields in intensity and deley difference stereophony]," Dissertation, Technische Hochschule, Aschen (1963).

[12] S. Amano, S. Sakamoto, T. Kondo and Y. Suzuki, "Development of familiarity-controlled word lists 2003 (FW03) to assess spoken-word intelligibility in Japanese," Speech Commun., 51, 76-82 (2009).

[13] J. Blauert, "An experiment in directional hearing with simultaneous optical stimulation," Acustica, 23, 118-119 (1970).

[14] M. B. Gardner, "Lateral localization of $0^{\circ}$ - or near- $0^{\circ}$-oriented speech signals in anechoic space," J. Acoust. Soc. Am., 44, 797-803 (1968).

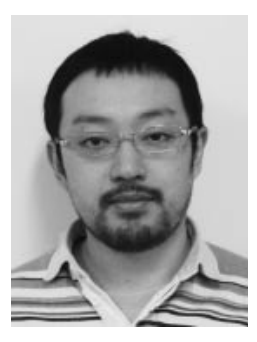

Koji Abe was graduated from Tohoku University in 1998, and received his $\mathrm{Ph}$. D. degree in 2003. He is currently an assistant professor at the Faculty of Systems Science and Technology, Akita Prefectural University. His main research interests are in multi-modal perception. He is a member of the Acoustical Society of Japan (ASJ), Japan Society of Kansei Engineering (JSKE).

Daisuke Sunada received the B. E. and M. E. degrees from Akita Prefectural University in 2003 and 2005, respectively.

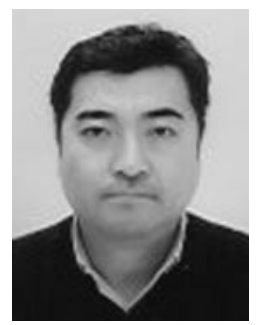

Shouichi Takane received his B. E., M. E., and $\mathrm{Ph}$. D. degrees from Tohoku University in 1989, 1991 and 1998, respectively. $\mathrm{He}$ is currently an associate professor at the Faculty of Systems Science and Technology, Akita Prefectural University. His research interests include high-definition acoustic display, sound field control, numerical analysis of sound field and acoustic signal processing. He is a member of the Acoustical Society of Japan (ASJ), the Institute of Electronics, Information and Communication Engineers (IEICE), the Virtual Reality Society of Japan (VRSJ), the Audio Engineering Society (AES), and other societies.

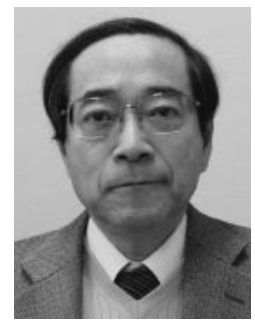

Sojun Sato was graduated from Tokyo Institute of Technology in 1970, and received his Doctor of Engineering degree in 1975. From 1977 to 2001, he joined the Electrotechnical Laboratory, where he researched precise acoustical measurements. From 2001 to 2006, he was the Head of the Acoustics and Vibration Metrology Division, National Metrology Institute of Japan, National Institute of Advanced Industrial Science and Technology. He is now a professor of the Faculty of Systems Science and Technology, Akita Prefectural University. He has been engaged in standardization work with ISO and IEC. He received the 20th Sato Paper Award from the ASJ. He is a member of ASJ, INCE/J, SICE, IEEE and some other academic societies. 